\title{
FGFR3 mutations in seborrheic keratoses are already present in flat lesions and associated with age and localization
}

\author{
Christian Hafner ${ }^{1}$, Arndt Hartmann ${ }^{2}$, Johanna MM van Oers ${ }^{3}$, Robert Stoehr ${ }^{4}$, \\ Ellen C Zwarthoff ${ }^{3}$, Ferdinand Hofstaedter ${ }^{2}$, Michael Landthaler ${ }^{1}$ and Thomas Vogt $^{1}$ \\ ${ }^{1}$ Department of Dermatology, University of Regensburg, Regensburg, Germany; ${ }^{2}$ Institute of Pathology, \\ University of Regensburg, Regensburg, Germany; ${ }^{3}$ Department of Pathology, Josephine Nefkens Institute, \\ Erasmus MC, CA Rotterdam, The Netherlands and ${ }^{4}$ Department of Urology, University of Regensburg, \\ Regensburg, Germany
}

\begin{abstract}
Somatic activating fibroblast growth factor 3 (FGFR3) mutations in human skin can cause seborrheic keratoses, one of the most frequent skin tumors in man. However, details of the involved mechanisms remain elusive. We analyzed 65 acanthotic seborrheic keratoses with varying vertical diameters for FGFR3 mutations using a SNaPshot ${ }^{\mathbb{R}}$ multiplex assay. Immunohistochemistry was performed for Ki-67, bcl-2 and FGFR3 protein in all seborrheic keratoses and 19 normal skin samples. FGFR3 mutations were detected in 37 of 65 seborrheic keratoses (57\%). These mutations were found both in flat (initial) and thick seborrheic keratoses. FGFR3 mutations were significantly associated with increased age and localization on the head and neck $(\boldsymbol{P}<0.01)$. Ki67 expression was significantly higher in seborrheic keratoses than in normal epidermis independent of the FGFR3 status $(P<0.001)$. Furthermore, FGFR3 mutations were associated with an increased expression of bcl-2 and FGFR3 protein $(P<0.05)$. Our results indicate that FGFR3 mutations can occur early in the pathogenesis of at least a subset of seborrheic keratoses. Increased age appears to be a risk factor for these mutations. The preferential occurrence of FGFR3 mutations in seborrheic keratoses of the head and neck suggests a causative role for cumulative lifetime ultraviolet light exposure.
\end{abstract}

Modern Pathology (2007) 20, 895-903; doi:10.1038/modpathol.3800837; published online 22 June 2007

Keywords: seborrheic keratosis; acanthosis; FGFR3 mutation; bcl-2; Ki-67

Seborrheic keratoses represent one of the most common skin tumors in man. Their prevalence increases with age. Seborrheic keratoses are detected in $80-100 \%$ of people over 50 years. ${ }^{1,2}$ Affected individuals can show large numbers of lesions. Yeatman et al reported an average number of 69 seborrheic keratoses/person in people aged more than 75 years. Seborrheic keratoses are typically localized on the head, the trunk and the extremities with the exception of the palms and soles. They present as well-demarcated brownish plaques and later may show a verrucous surface. Their horizontal diameter ranges from a few millimeters to several centimeters. Three major histologic subtypes are known including acanthotic, hyperkeratotic and

Correspondence: Dr C Hafner, MD, Department of Dermatology, University of Regensburg, Franz-Josef-Strauss-Allee 11, 93042 Regensburg, Germany.

E-mail: christian.hafner@klinik.uni-regensburg.de

Received 26 March 2007; revised 10 May 2007; accepted 17 May 2007; published online 22 June 2007 adenoid seborrheic keratoses variants. ${ }^{3}$ Common histological features are acanthosis, papillomatosis and hyperkeratosis along with a varying degree of pigmentation. The vertical diameter (tumor thickness) of seborrheic keratoses varies considerably. Flat (initial) seborrheic keratoses frequently show gradual vertical growth within years. ${ }^{3}$ However, malignant transformation is a very rare event in seborrheic keratoses, and the reported cases may also represent collision tumors of seborrheic keratoses and other epidermal malignancies such as basal cell carcinoma and squamous cell carcinoma. ${ }^{4}$

Although seborrheic keratoses are very common tumors and represent one of the most disfiguring signs of skin aging, their pathogenesis is only marginally understood. Recently, activating FGFR3 mutations in the epidermis were shown to be involved in the development of seborrheic keratoses. ${ }^{5}$ Transgenic mice expressing the S249C FGFR3 mutation in the basal layer of the epidermis under the control of the keratin 5 promoter developed thickening of the skin and verrucous skin tumors 
with histological features similar to human seborrheic keratoses. FGFR3 mutations have been identified in $39-85 \%$ of human seborrheic keratoses. ${ }^{5,6}$ In patients with FGFR3 mutant seborrheic keratoses, normal skin showed a wild-type FGFR3 sequence confirming the somatic nature of these mutations. Albeit an important role of FGFR3 mutations in the pathogenesis of seborrheic keratoses is suggested by previous studies, details of the mechanisms causing these mutations in human epidermis and of the signaling pathways mediating the growth of acanthotic tumors in FGFR3 mutant skin are unknown. Therefore, we analyzed 65 acanthotic seborrheic keratoses for the presence of FGFR3 mutations. The FGFR3 status was correlated with the thickness of the seborrheic keratoses to determine whether FGFR3 mutations are early or late genetic alterations in the pathogenesis of seborrheic keratoses. In addition, the FGFR3 status was correlated with Ki-67, bcl-2 and FGFR3 protein expression to investigate whether increased proliferation, anti-apoptotic mechanisms and FGFR3 protein overexpression are associated with FGFR3 mutations in human seborrheic keratoses.

\section{Materials and methods}

\section{Patient Material and Microdissection}

Sixty-five histologically confirmed acanthotic seborrheic keratoses of 65 individuals (33 men and 32 women) were retrieved from the histology files of the Department of Dermatology, University of Regensburg. The characteristics of the persons are shown in Table 1. Nineteen normal skin samples from resection margins served as controls for immunohistochemical analyses. From two patients with seborrheic keratoses, normal skin adjacent to the seborrheic keratoses was additionally available. Informed consent had been obtained from all patients according to the guidelines of the local ethics committee and the declaration of Helsinki. The vertical diameter (thickness) of each acanthotic seborrheic keratosis was measured on an H\&E stained reference section using a scale bar. Furthermore, sections of $10 \mu \mathrm{m}$ thickness from formalinfixed and paraffin-embedded seborrheic keratoses tissues were microdissected manually with a needle under an inverted microscope to obtain at least $80 \%$ seborrheic keratoses cells.

\section{DNA Isolation}

Isolation of DNA was performed following standard protocols. In brief, about $10-50 \mathrm{mg}$ of dissected tissue was digested with proteinase $\mathrm{K}$ overnight in lysis buffer, and DNA was isolated with the Magna Pure ${ }^{\circledR}$ PCR Template Preparation Kit (Roche Diagnostics) according to the manufacturer's protocol.

\section{SNaPshot ${ }^{\circledR}$ Multiplex Assay}

We used a previously described high-throughput SnaPshot multiplex mutation detection assay for the screening of 11 activating FGFR3 point mutations. ${ }^{7,8}$ In brief, three regions of interest in exons 7, 10 and 15 comprising known FGFR3 point mutations were amplified in one multiplex polymerase chain reaction. Mutation-specific primers were then extended with labeled di-deoxynucleotides. The SNaPshot multiplex assay covers the following 11 FGFR3 point mutations: c.742C $>\mathrm{T}$ (R248C), c.746C $>\mathrm{G}$ (S249C), c.1114G $>$ T (G372C), c.1117A $>$ T (S373C), c.1124A > G (Y375C), c.1144G > A (G382R), c.1178C > A (A393E), c.1954A $>$ G (K652E), c.1954A $>$ C (K652Q), c.1955A > T (K652M), c.1955A >C (K652T). The nucleotide positions correspond to the coding sequence of the FGFR3 IIIb isoform, where position 1 is the A of the ATG. FGFR3 IIIb is the predominantly expressed isoform in epithelial cells such as keratinocytes. The assay covered all yet-identified FGFR3 mutations in benign acanthotic skin tumors (seborrheic keratoses, epidermal nevi) and urothelial carcinoma. The presence or absence of a mutation was indicated by the incorporated wild type or mutant labeled di-deoxynucleotide. Each mutation was confirmed by an independent reaction.

\section{Immunohistochemistry}

Immunohistochemical analysis was performed in 65 acanthotic seborrheic keratoses and 19 normal skin samples for the analysis of Ki-67, bcl-2 and FGFR3 protein expression. Sections of paraffin-embedded tissues with a thickness of $1.5 \mu \mathrm{m}$ were deparaffinized, rehydrated and blocked with $3 \% \mathrm{H}_{2} \mathrm{O}_{2} / 70 \%$ $\mathrm{CH}_{3} \mathrm{OH}$ for $10 \mathrm{~min}$. They were incubated in citrate buffer $(\mathrm{pH} 6.0)$ at $90^{\circ} \mathrm{C}$ for $20 \mathrm{~min}$. Then the samples were incubated with the primary antibody dilution at room temperature for one hour (Ki-67 1:100, bcl-2 1:50, FGFR3 1:50). Antibodies were purchased from Dako (Ki-67, bcl-2) and Santa Cruz (FGFR3). Staining was performed using the Zyto Chem Plus HRP Broad Spectrum Kit (Zytomed, Berlin) according to the manufacturer's protocol. Negative controls were performed by omitting the primary antibody. Evaluation of the protein expression was performed without knowledge of the FGFR3 mutational status of the samples. Ki-67positive cells were counted in five representative areas of each section, and at least 200 nuclei were analyzed in each of these areas. The percentage of Ki-67-positive cells was then calculated for each section. Immunohistochemical staining for bcl-2 and FGFR3 was evaluated using a semiquantitative score: 0 (not expressed), 1 (positive staining of about $1-30 \%$ of cells), 2 (positive staining of about $31-$ $60 \%$ of cells) and 3 (positive staining of more than $60 \%$ of cells). 
Table 1 Analysis of 65 acanthotic seborrheic keratoses

\begin{tabular}{|c|c|c|c|c|c|c|c|c|}
\hline \multirow[t]{2}{*}{ No. } & \multirow[t]{2}{*}{ Sex } & \multirow[t]{2}{*}{ Age } & \multirow[t]{2}{*}{ Localization } & \multirow[t]{2}{*}{ Thickness (mm) } & \multirow{2}{*}{$\begin{array}{l}\text { FGFR3 mutation } \\
\text { analysis }\end{array}$} & \multicolumn{3}{|c|}{ Immunohistochemistry } \\
\hline & & & & & & $K i-67(\%)$ & $b c l-2$ & FGFR3 \\
\hline 1 & $\mathrm{~m}$ & 75 & Trunk & 0.13 & wt & 4.8 & ++ & ++ \\
\hline 2 & $\mathrm{~m}$ & 41 & Head & 0.15 & wt & 3.4 & ++ & + \\
\hline 3 & $\mathrm{~m}$ & 45 & Head & 0.28 & K652M & 6.9 & ++ & ++ \\
\hline 4 & f & 72 & Trunk & 0.42 & wt & 3.4 & + & + \\
\hline 5 & $\mathrm{f}$ & 57 & Trunk & 0.36 & S373C & 11.6 & + & + \\
\hline 6 & $\mathrm{~m}$ & 64 & Head & 0.28 & R248C & 5.2 & +++ & + \\
\hline 7 & f & 64 & Trunk & 0.10 & S249C & 3.0 & ++ & + \\
\hline 8 & $\mathrm{~m}$ & 49 & Trunk & 0.25 & wt & 4.0 & + & + \\
\hline 9 & f & 67 & Arm & 0.35 & wt & 8.5 & + & ++ \\
\hline 10 & $\mathrm{f}$ & 48 & Trunk & 0.72 & wt & 18.1 & +++ & ++ \\
\hline 11 & $\mathrm{f}$ & 56 & Trunk & 0.10 & wt & 6.4 & + & + \\
\hline 12 & $\mathrm{f}$ & 52 & Neck & 0.43 & K652M & 3.5 & ++ & + \\
\hline 13 & $\mathrm{~m}$ & 59 & Head & 0.37 & Y375C & 5.1 & ++ & + \\
\hline 14 & f & 51 & Trunk & 0.13 & wt & 2.5 & ++ & ++ \\
\hline 15 & $\mathrm{f}$ & 70 & Neck & 1.35 & K652M & 3.4 & +++ & ++ \\
\hline 16 & $\mathrm{~m}$ & 63 & Trunk & 0.10 & wt & 3.5 & + & + \\
\hline 17 & f & 61 & Trunk & 1.30 & K652M & 10.7 & ++ & ++ \\
\hline 18 & $\mathrm{~m}$ & 61 & Head & 1.10 & G372C & 9.5 & +++ & ++ \\
\hline 19 & f & 49 & Trunk & 1.00 & Y375C & 7.6 & ++ & ++ \\
\hline 20 & $\mathrm{~m}$ & 78 & Trunk & 0.47 & K652M & 3.0 & ++ & ++ \\
\hline 21 & $\mathrm{~m}$ & 78 & Head & 0.55 & wt & 6.0 & ++ & + \\
\hline 22 & $\mathrm{f}$ & 74 & Trunk & 1.00 & K652E & 7.9 & + & + \\
\hline 23 & $\mathrm{f}$ & 67 & Trunk & 1.10 & wt & 8.1 & + & + \\
\hline 24 & $\mathrm{~m}$ & 56 & Head & 1.05 & S249C & 9.4 & ++ & ++ \\
\hline 25 & $\mathrm{~m}$ & 64 & Trunk & 0.85 & K652E & 5.9 & + & + \\
\hline 26 & f & 84 & Head & 1.30 & K652M & 3.4 & +++ & ++ \\
\hline 27 & $\mathrm{~m}$ & 68 & Trunk & 1.20 & K652M & 4.3 & ++ & ++ \\
\hline 28 & $\mathrm{~m}$ & 60 & Neck & 1.30 & wt & 1.8 & ++ & +++ \\
\hline 29 & $\mathrm{~m}$ & 65 & Trunk & 0.52 & R248C & 8.9 & +++ & ++ \\
\hline 30 & f & 52 & Trunk & 1.20 & K652M & 1.5 & + & ++ \\
\hline 31 & $\mathrm{f}$ & 82 & Head & 0.65 & R248C & 6.4 & + & ++ \\
\hline 32 & $\mathrm{~m}$ & 75 & Trunk & 0.38 & wt & 5.0 & +++ & ++ \\
\hline 33 & $\mathrm{~m}$ & 67 & Head & 1.30 & R248C & 14.9 & + & ++ \\
\hline 34 & $\mathrm{f}$ & 66 & Thigh & 0.90 & wt & 10.2 & ++ & ++ \\
\hline 35 & $\mathrm{f}$ & 67 & Neck & 0.72 & Y375C & 3.8 & +++ & ++ \\
\hline 36 & $\mathrm{~m}$ & 62 & Head & 1.10 & R248C & 4.6 & + & + \\
\hline 37 & $\mathrm{f}$ & 71 & Head & 0.94 & $\mathrm{R} 248 \mathrm{C}$ & 5.1 & ++ & ++ \\
\hline 38 & $\mathrm{~m}$ & 44 & Trunk & 1.00 & wt & 5.4 & ++ & ++ \\
\hline 39 & f & 55 & Neck & 2.05 & G372C & 7.4 & + & ++ \\
\hline 40 & $\mathrm{~m}$ & 66 & Head & 0.90 & S249C & 6.5 & +++ & +++ \\
\hline 41 & $\mathrm{~m}$ & 63 & Trunk & 0.70 & wt & 6.5 & + & + \\
\hline 42 & $\mathrm{~m}$ & 77 & Trunk & 0.60 & wt & 3.2 & + & + \\
\hline 43 & f & 66 & Head & 0.80 & R248C & 2.8 & ++ & ++ \\
\hline 44 & $\mathrm{f}$ & 34 & Trunk & 0.65 & wt & 11.0 & + & +++ \\
\hline 45 & $\mathrm{~m}$ & 70 & Trunk & 0.50 & Y375C & 6.6 & - & + \\
\hline 46 & $\mathrm{~m}$ & 67 & Head & 1.25 & R248C & 12.5 & ++ & +++ \\
\hline 47 & f & 44 & Head & 0.20 & wt & 10.5 & + & + \\
\hline 48 & $\mathrm{f}$ & 70 & Head & 1.30 & R248C & 14.2 & ++ & +++ \\
\hline 49 & $\mathrm{~m}$ & 39 & Trunk & 0.80 & wt & 8.8 & + & + \\
\hline 50 & $\mathrm{~m}$ & 37 & Head & 3.20 & S373C & 8.8 & ++ & +++ \\
\hline 51 & f & 82 & Trunk & 0.40 & R248C & 4.5 & ++ & + \\
\hline 52 & $\mathrm{f}$ & 83 & Head & 0.70 & R248C & 6.5 & ++ & +++ \\
\hline 53 & $\mathrm{f}$ & 26 & Trunk & 0.70 & wt & 11.5 & + & ++ \\
\hline 54 & $\mathrm{f}$ & 59 & Trunk & 0.75 & wt & 7.2 & +++ & +++ \\
\hline 55 & $\mathrm{f}$ & 51 & Trunk & 1.35 & R248C & 6.4 & +++ & +++ \\
\hline 56 & $\mathrm{~m}$ & 45 & Trunk & 2.40 & wt & 12.7 & + & + \\
\hline 57 & $\mathrm{~m}$ & 25 & Trunk & 1.20 & wt & 12.0 & + & + \\
\hline 58 & f & 59 & Trunk & 1.60 & wt & 14.1 & + & + \\
\hline 59 & $\mathrm{~m}$ & 50 & Trunk & 1.40 & wt & 4.3 & ++ & + \\
\hline 60 & $\mathrm{f}$ & 60 & Head & 1.10 & wt & 10.0 & ++ & + \\
\hline 61 & $\mathrm{~m}$ & 59 & Trunk & 1.20 & R248C & 9.2 & +++ & +++ \\
\hline 62 & $\mathrm{~m}$ & 63 & Head & 1.10 & R248C & 7.8 & + & + \\
\hline 63 & f & 76 & Head & 0.20 & K652M & 4.9 & ++ & ++ \\
\hline 64 & $\mathrm{~m}$ & 33 & Neck & 0.30 & wt & 11.3 & + & +++ \\
\hline 65 & $\mathrm{~m}$ & 64 & Trunk & 0.70 & G372C & 9.1 & ++ & +++ \\
\hline
\end{tabular}

$\mathrm{m}$, male; f, female; age, age at the time of the seborrheic keratoses removal; maximal thickness of acanthotic seborrheic keratoses was measured using a scale bar on an H\&E-stained section of each sample; FGFR3 mutation analysis was performed with a multiplex SNaPshot ${ }^{\mathbb{B}}$ assay covering 11 known activating FGFR3 mutations (R248C, S249C, G372C, S373C, Y375C, G382R, A393E, K652E, K652 M, K652Q and K652T; codons are numbered according to the open reading frame of the FGFR3b isoform, which is predominantly found in epithelial cells); Ki-67 expression was assessed immunohistochemically by counting the proportion of Ki-67-positive cells in five representative areas (at least 200 cells/area) of each section; bcl-2 and FGFR3 protein expression were detected immunohistochemically and scored from $(-)=$ negative to $(+++)=$ strong positive. 


\section{Statistical Analysis}

The non-parametric Mann-Whitney $U$-test was used for statistical analysis. In addition, the cross-tab test was used for the analysis of the association between FGFR3 status and the localization of seborrheic keratoses. A $P$-value $<0.05$ was considered significant.

\section{Results}

Analysis of FGFR3 Mutations in Acanthotic Seborrheic Keratoses

FGFR3 mutations were found in 37 of 65 seborrheic keratoses (57\%), while 28 of 65 seborrheic keratoses

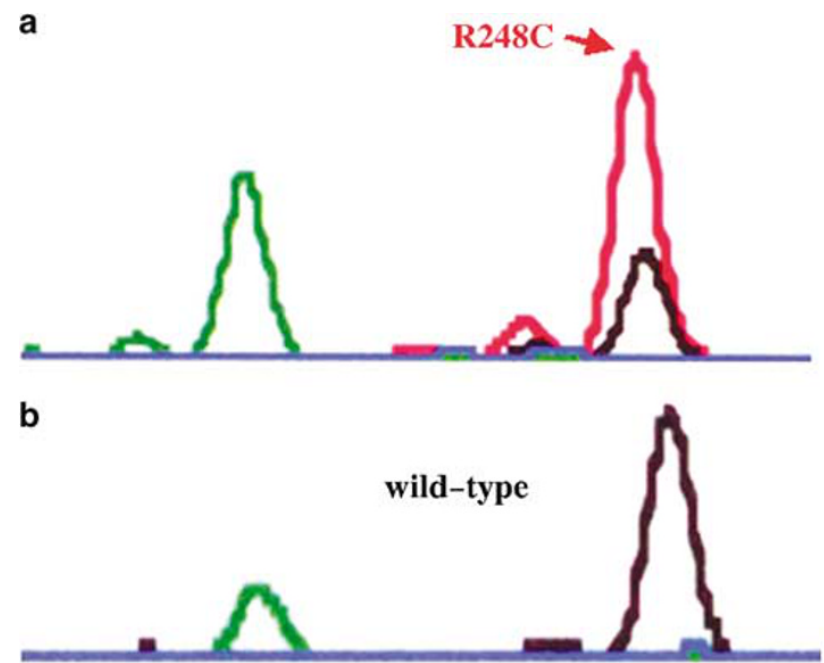

C

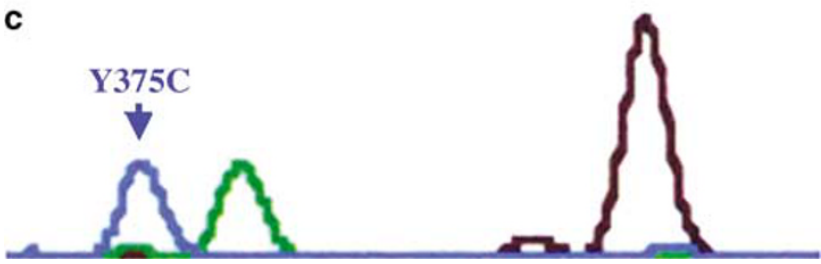

Figure $1 \mathrm{SNaPshot}$ analysis of human seborrheic keratoses. (a) The R248C mutation was the most frequent FGFR3 mutation detected in this study (patient no. 55). (b) Normal epidermis adjacent to the seborrheic keratoses in patient no. 45 showed a wild-type FGFR3 status, confirming the somatic nature of FGFR3 mutations. (c) The seborrheic keratoses of patient no. 45 revealed a Y375C mutation.
$(43 \%)$ revealed a wild-type status at the investigated loci (Table 1). The most frequent mutation was R248C (Figure 1a). This mutation was detected in 14 of 37 seborrheic keratoses (38\%). The numbers of seborrheic keratoses revealing other FGFR3 mutations were $n=9(24 \%)$ for K652M, $n=4(11 \%)$ for Y375C, $n=3(8 \%)$ for S249C, $n=3(8 \%)$ for G372C, $n=2(5 \%)$ for S373C and $n=2(5 \%)$ for K652E (Table 2). Neither the A393E mutation nor double mutations, which both had been described previously in seborrheic keratoses, ${ }^{6}$ were observed in this study. From two individuals (no. 44 and 45), additional normal skin adjacent to the seborrheic keratoses was available for mutation analysis. Both normal skin samples displayed a wild-type FGFR3 sequence (Figure 1b), while the seborrheic keratoses of patient 45 showed a Y375C mutation (Figure 1c). This finding confirms the somatic nature of FGFR3 mutations in human seborrheic keratoses.

\section{FGFR3 Mutations are already Present in Flat Seborrheic Keratoses}

The thickness of acanthotic seborrheic keratoses varies considerably. Dermatologists clinically observe flat lesions with a mild acanthosis in histopathology as well as thick and verrucous seborrheic keratoses, histologically showing a marked acanthosis. The flat lesions are often referred to as initial seborrheic keratoses, which can subsequently proceed to thick seborrheic keratoses. ${ }^{3}$ We sought to investigate whether FGFR3 mutations are already present in flat (initial) seborrheic keratoses and therefore may represent early genetic alterations in the pathogenesis of seborrheic keratoses, or whether FGFR3 mutations are associated with more prominent acanthosis. The latter would indicate that these mutations occur later in the pathogenesis of seborrheic keratoses and promote vertical growth of the flat seborrheic keratoses.

To test these two hypotheses, we measured the thickness of the acanthotic seborrheic keratoses in our series (Figure 2a). The mean thickness of all seborrheic keratoses was $0.84 \pm 0.56 \mathrm{~mm}$ (range: $0.10-3.20 \mathrm{~mm}$ ). Acanthotic seborrheic keratoses showing a wild-type sequence in the SNaPshot

Table 2 FGFR3 mutations in 65 acanthotic seborrheic keratoses

\begin{tabular}{|c|c|c|c|c|c|}
\hline FGFR3 mutation & Number of SK & $\%$ of mutations & Nucleotide position & Predicted effect & Germline $^{a}$ \\
\hline R248C & 14 & 37.8 & c. $742 \mathrm{C}>\mathrm{T}$ & p.Arg248Cys & TD I \\
\hline K652M & 9 & 24.3 & c. $1955 \mathrm{~A}>\mathrm{T}$ & p.Lys652Met & SADDAN \\
\hline Y375C & 4 & 10.8 & c. $1124 \mathrm{~A}>\mathrm{G}$ & p.Tyr375Cys & TD I \\
\hline S249C & 3 & 8.1 & c. $746 \mathrm{C}>\mathrm{G}$ & p.Ser249Cys & TD I \\
\hline G372C & 3 & 8.1 & c. $1114 \mathrm{G}>\mathrm{T}$ & p.Gly372Cys & TD I \\
\hline S373C & 2 & 5.4 & c. $1117 \mathrm{~A}>\mathrm{T}$ & p.Ser373Cys & TD I \\
\hline K652E & 2 & 5.4 & c. $1954 A>G$ & p.Lys652Glu & TD II \\
\hline
\end{tabular}

SK, seborrheic keratosis.

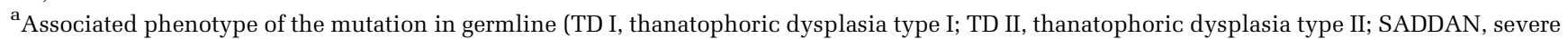
achondroplasia with developmental delay and acanthosis nigricans). 
a
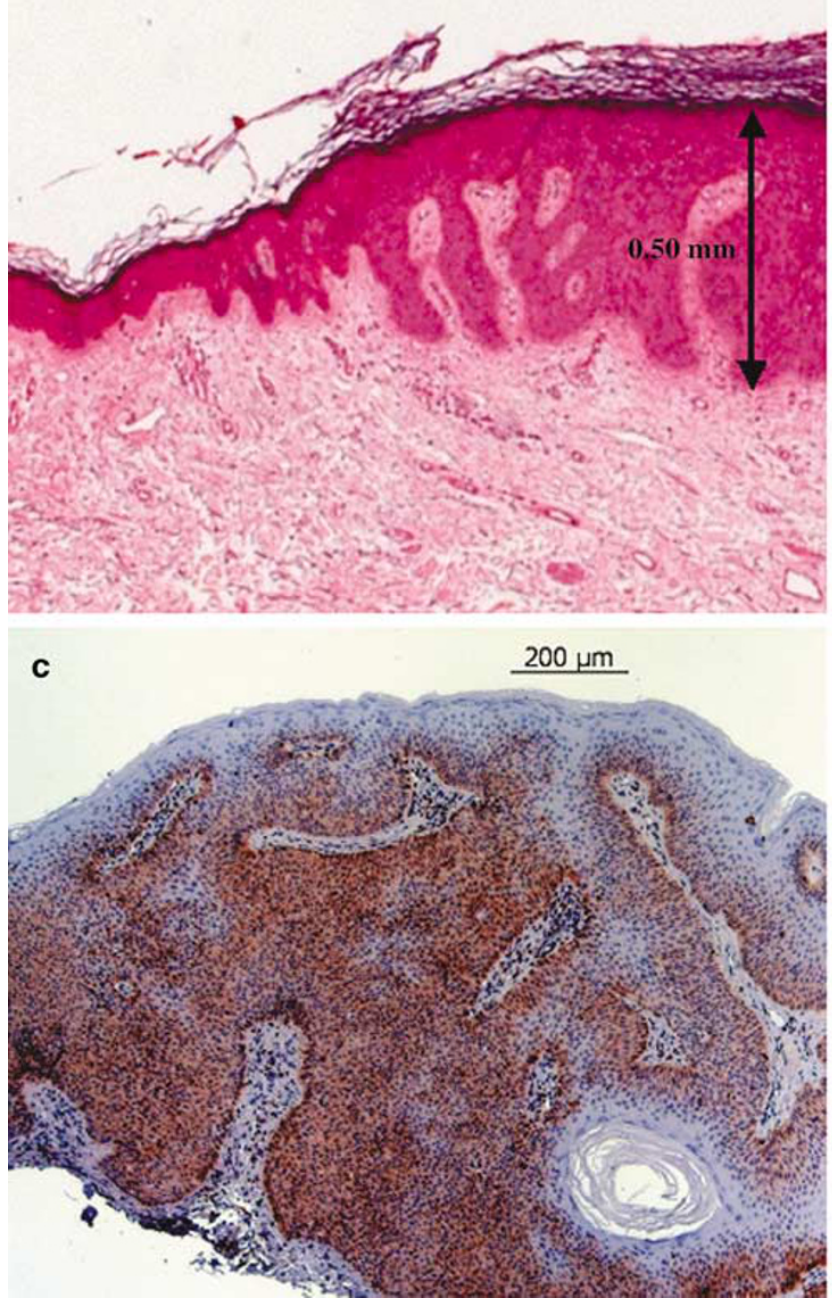

b.

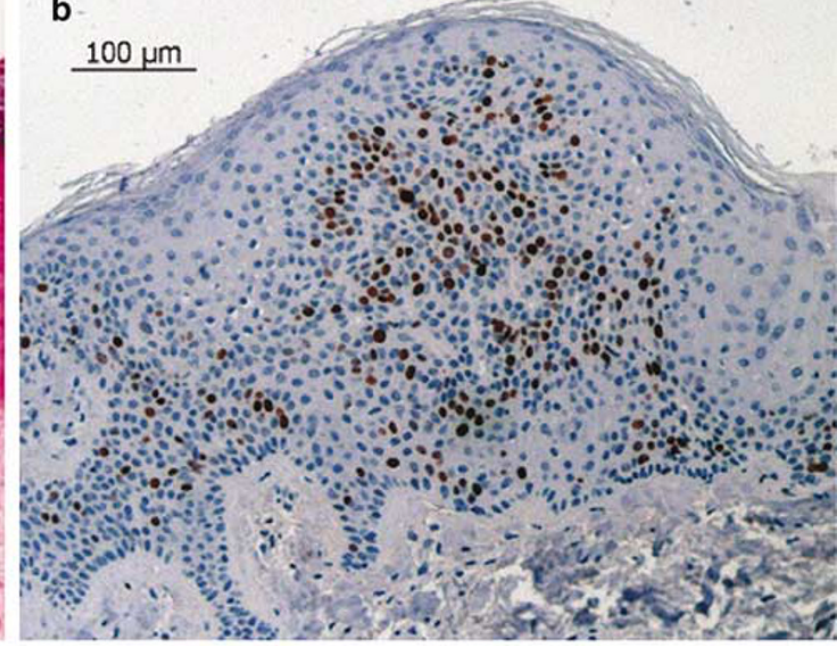

d $\quad 100 \mu \mathrm{m}$

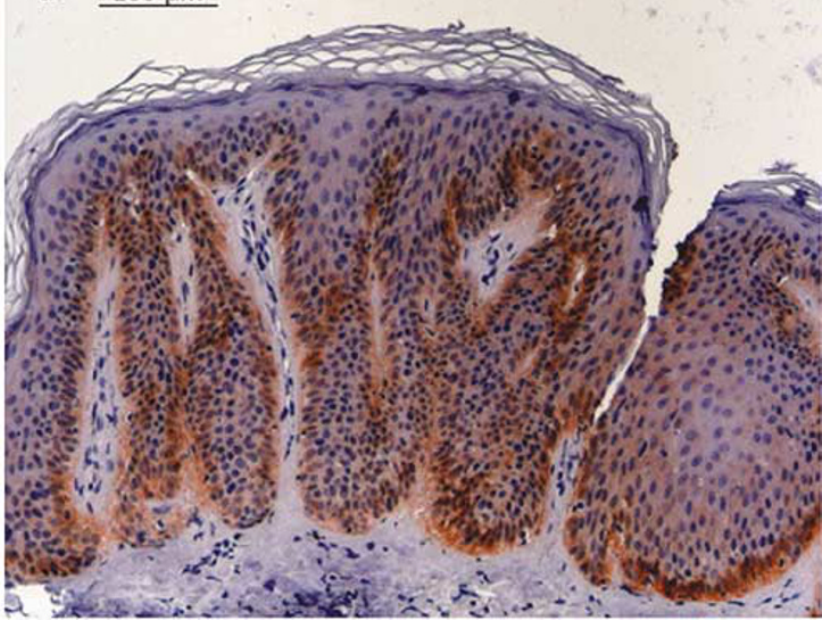

Figure 2 Immunohistochemical analysis of acanthotic seborrheic keratoses. (a) The vertical diameter of the acanthotic seborrheic keratoses was measured on H\&E stained sections (patient no. 45). (b) Immunohistochemical staining for Ki-67 antigen revealed an increased proliferation rate in seborrheic keratoses compared with normal epidermis (patient no. 60). (c) Immunohistochemical staining for bcl-2 antigen in seborrheic keratoses was significantly higher in FGFR3 mutant seborrheic keratoses (patient no. 15). (d) Immunohistochemical staining for FGFR3 protein showed FGFR3 protein expression in all seborrheic keratoses with a preferential staining of the basal epidermis in most samples (patient no. 31).

analysis showed a mean thickness of $0.71 \pm$ $0.53 \mathrm{~mm}$ (Figure 3). The mean thickness of seborrheic keratoses with FGFR3 mutations was $0.93 \pm$ $0.56 \mathrm{~mm}$. Although FGFR3 mutant seborrheic keratoses showed a slightly increased mean thickness compared with wild-type seborrheic keratoses, the difference between wild type and mutant seborrheic keratoses was not significant $(P=0.07)$. Our results indicate that FGFR3 mutations occur both in flat and thick seborrheic keratoses. Therefore, FGFR3 mutations are early genetic events in the pathogenesis of at least a subset of seborrheic keratoses.

\section{FGFR3 Mutations in Human Seborrheic Keratoses are Associated with Age and Localization}

The age of the analyzed patients ranged from 25 to 84 years (mean age: $60.1 \pm 13.6$ years; median age: 63 years). Individuals with FGFR3 mutant seborrheic keratoses were significantly older than individuals with wild-type seborrheic keratoses (Figure 4). The mean age of individuals with wild-type seborrheic keratoses was $54.5 \pm 15.0$ years, while the mean age of the individuals with FGFR3 mutant seborrheic keratoses was $64.4 \pm 10.5$ years $(P=0.009)$. Our results suggest that the frequency of the investigated FGFR3 mutations in human seborrheic keratoses may increase with age. By contrast, there was no significant correlation between the sex and FGFR3 mutations in seborrheic keratoses $(P=0.70)$.

The analyzed seborrheic keratoses of this study were localized on the head (22), neck (6), trunk (35), arm (1) and thigh (1). We investigated whether the FGFR3 status of seborrheic keratoses is different in chronically UV-light exposed skin (head and neck) versus in skin with intermittent UV-light exposure (trunk, arm and thigh) (Figure 5). Interestingly, 18 


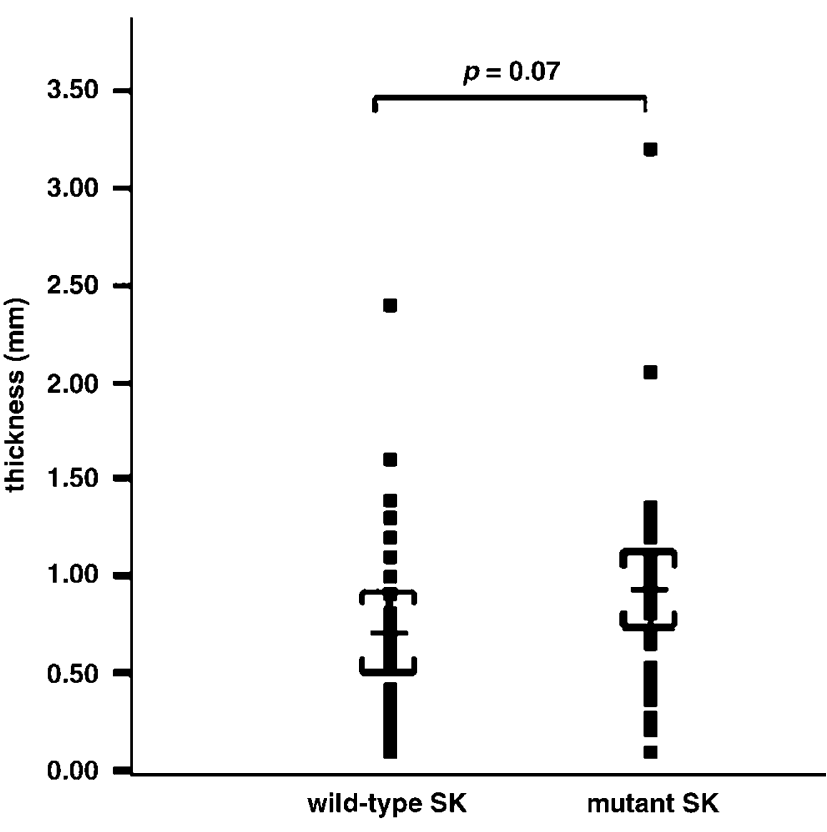

Figure 3 The average thickness was not significantly different in FGFR3 wild-type and mutant seborrheic keratoses, indicating that FGFR3 mutations occur both in flat and thick seborrheic keratoses. Thus, FGFR3 mutations appear to be early genetic events in the pathogenesis of at least a subset of seborrheic keratoses (mean values and 95\% confidence intervals of the mean values are indicated).

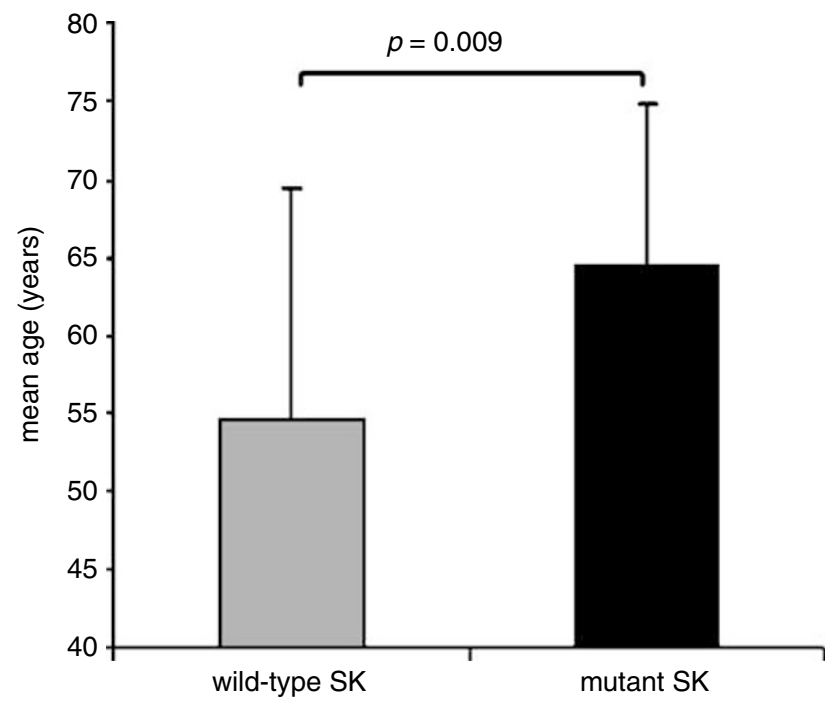

Figure 4 The mean age of individuals with FGFR3 mutant seborrheic keratoses was significantly higher than that of individuals with wild-type seborrheic keratoses.

of 22 seborrheic keratoses (82\%) localized on the head revealed an FGFR3 mutation, while 15 of 35 seborrheic keratoses (43\%) of the trunk showed a mutant FGFR3. Seborrheic keratoses of the neck harboured FGFR3 mutations in four of six cases (67\%). Seborrheic keratoses of the head and neck revealed significantly more FGFR3 mutations than seborrheic keratoses of the trunk and the extremities

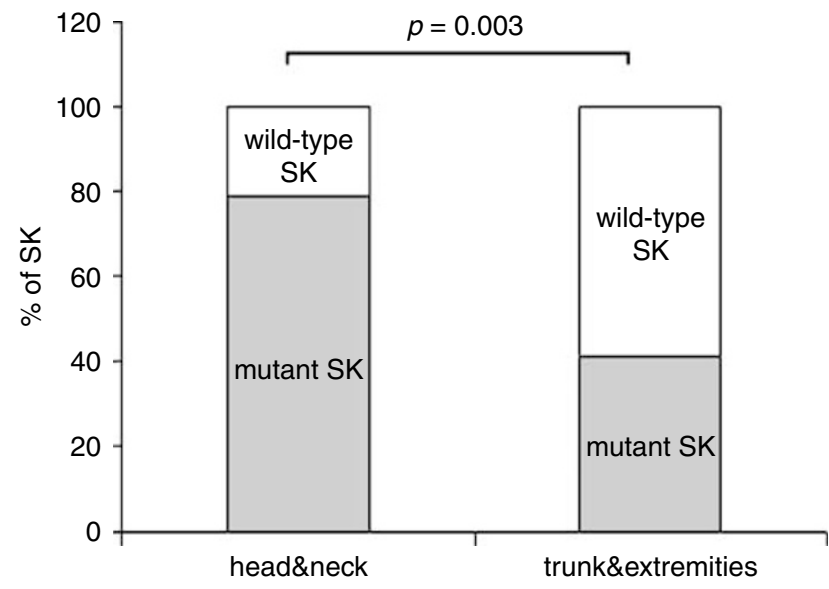

Figure 5 Seborrheic keratoses localized on the head and neck showed a significant higher frequency of FGFR3 mutations than seborrheic keratoses on the trunk and extremities.

$(P=0.003)$. The cumulative life-time UV-light exposure of the head and neck region is assumed to be higher than that of the trunk, suggesting that the increased rate of FGFR3 mutations at these regions may be linked to UV-light exposure. The R248C mutation, the most frequent mutation in this study, represents a typical UV-signature mutation with a $\mathrm{C} \rightarrow \mathrm{T}$ transition at a dipyrimidine site. Accordingly, the proportion of seborrheic keratoses of the head and neck region revealing a R248C mutation was $46 \%$ (10 of 22 seborrheic keratoses) and therefore higher than the proportion of this mutation in seborrheic keratoses of the trunk and extremities (4 of 15 seborrheic keratoses; $27 \%$ ), but this was not significant $(P=0.25)$.

\section{Seborrheic Keratoses Show Increased Ki-67 Expression Independent of FGFR3 Status}

The mechanisms leading to the development of acanthosis, papillomatosis, hyperkeratosis and hyperpigmentation in FGFR3 mutant skin and details of the involved signaling pathways downstream of the mutant FGFR3 protein in the epidermis are largely unknown. We performed immunohistochemistry for Ki-67 protein to assess a possible contribution of hyperproliferation to the development of human seborrheic keratoses and its association with FGFR3 mutations (Figure 2b). We additionally analyzed 19 normal skin samples to compare the protein expression of seborrheic keratoses and normal epidermis.

In FGFR3 wild-type seborrheic keratoses, $7.7 \pm$ $4.0 \%$ of the cells were Ki-67 positive, and in FGFR3 mutant seborrheic keratoses $6.8 \pm 3.1 \%$ (Figure 6). $\mathrm{Ki}-67$ expression as a marker for proliferation was not significantly different in FGFR3 wild-type and mutant seborrheic keratoses $(P=0.53)$. However, the mean Ki-67 expression in normal epidermis was $2.3 \pm 0.8 \%$ and therefore significantly lower than in 


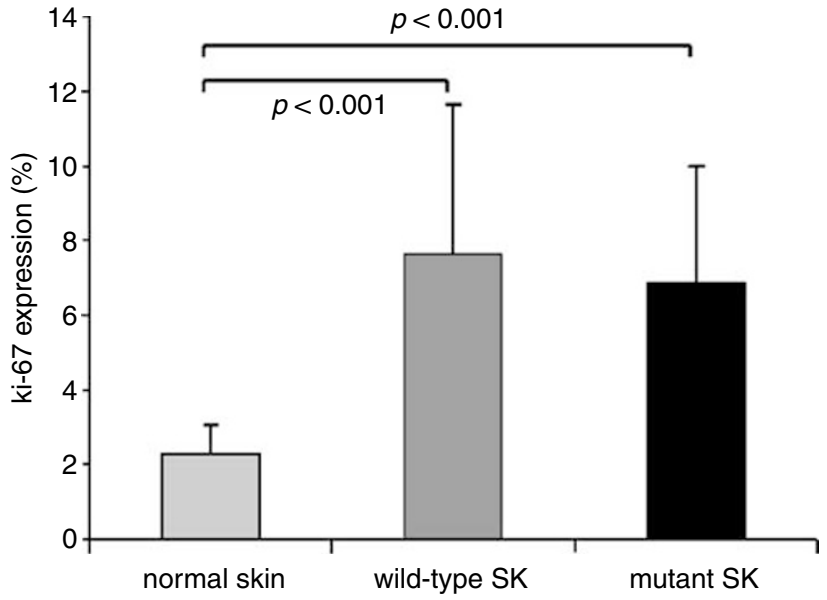

Figure $6 \mathrm{Ki}-67$ expression as a marker of proliferation was significantly increased in seborrheic keratoses compared with normal epidermis independent of their FGFR3 status.

seborrheic keratoses $(P<0.001)$. These results indicate that Ki-67 expression is increased in the epidermis of human seborrheic keratoses compared with normal epidermis independent of the FGFR3 status. Remarkably, both seborrheic keratoses groups (wild type and mutant) showed a considerable variance regarding Ki-67 expression (range: 1.5$18 \%)$.

\section{FGFR3 Mutations are Associated with Bcl-2 Protein Expression}

Resistance to apoptosis of keratinocytes could be another mechanism for the development of acanthosis in seborrheic keratoses. We therefore stained 65 seborrheic keratoses and 19 normal skin samples for the expression of anti-apoptotic bcl-2 protein (Figure 2c). Bcl-2 was predominantly expressed in the basal layers of the epidermis both in normal epidermis and seborrheic keratoses as reported previously. ${ }^{9}$ However, some control and seborrheic keratoses samples additionally revealed bcl-2 expression in suprabasal layers of the epidermis. The bcl-2 expression was scored from 0 (negative) to 3 (strong positive). The mean bcl-2 expression score was $1.6 \pm 0.7$ in normal epidermis, and $1.5 \pm 0.7$ in wild-type seborrheic keratoses. By contrast, seborrheic keratoses with FGFR3 mutations revealed a mean bcl-2 expression score of $2.0 \pm 0.8$. The difference of bcl-2 expression between wild type and mutant seborrheic keratoses was significant $(P=0.02)$. These results suggest a possible trend to an increased expression of anti-apoptotic bcl-2 protein in FGFR3 mutant seborrheic keratoses.

\section{Expression of FGFR3 Protein in Seborrheic Keratoses}

Since FGFR3 protein overexpression has been reported in other tumors and the expression in human seborrheic keratoses has not been analyzed yet, we also investigated FGFR3 protein expression in human seborrheic keratoses and control samples by immunohistochemistry. FGFR3 protein was preferentially expressed in the basal layer of the epidermis both in seborrheic keratoses and normal skin (Figure 2d), but some samples additionally showed suprabasal staining. The mean FGFR3 expression score was $1.6 \pm 0.7$ both in normal epidermis and in FGFR3 wild-type seborrheic keratoses, while the expression score of the FGFR3 mutant seborrheic keratoses was $1.9 \pm 0.7$ $(P=0.048)$. Immunohistochemistry shows that FGFR3 protein is regularly expressed in the basal epidermis of human seborrheic keratoses and may show a slightly increased expression in seborrheic keratoses with FGFR3 mutations.

\section{Discussion}

Although seborrheic keratoses are one of the most common skin tumors in man, their pathogenesis is still poorly understood. A causative role of somatic FGFR3 mutations in human seborrheic keratoses resulting in a constitutive ligand-independent receptor activation has been reported. ${ }^{5}$ Epidermal nevi, which are benign congenital skin lesions and histologically very similar to seborrheic keratoses, show also FGFR3 mutations as an underlying genetic basis. ${ }^{8}$ Therefore, evidence is growing that FGFR3 mutations are important genetic alterations in the pathogenesis of benign acanthotic skin tumors. ${ }^{10}$

However, not all seborrheic keratoses and epidermal nevi display FGFR3 mutations, suggesting the involvement of further genes. In the present study, we detected FGFR3 mutations in $57 \%$ of seborrheic keratoses, which is within the reported range from 26 to $85 \% .^{5,6,11}$ Most of the FGFR3 mutations in human skin tumors as well as in urothelial carcinoma and in the germline of patients with skeletal dysplasia syndromes appear to be restricted to several hot spots in exons 7,10 , and $15 .^{5,6,8,12-14}$ Interestingly, all FGFR3 mutations detected in human skin tumors so far are associated with thanatophoric dysplasia, SADDAN (severe achondroplasia with developmental delay and acanthosis nigricans) syndrome and Crouzon syndrome in germline. The FGFR3 mutations causing the above-mentioned skeletal dysplasia syndromes are associated with a strong constitutive receptor activation. ${ }^{15}$ Compatible with these observations, patients with thanatophoric dysplasia, SADDAN syndrome and Crouzon syndrome develop acanthosis nigricans of the skin, a condition closely resembling both seborrheic keratoses and epidermal nevi in histopathology. By contrast, the G382R mutation causing achondroplasia in germline and probably representing the nucleotide with the highest mutation frequency in the human genome, ${ }^{13}$ has not been found in seborrheic keratoses and only 
rarely in urothelial tumors. This mutation is linked with a less pronounced receptor activation, ${ }^{15}$ and achondroplasia patients usually do not show acanthosis nigricans of the skin. We have therefore hypothesized that the development of benign acanthotic skin tumors obviously requires a strong degree of FGFR3 activation. ${ }^{10}$ The data of the presented study further confirm this concept because the G382R mutation was not detected among the 65 seborrheic keratoses.

While an involvement of FGFR3 mutations in the pathogenesis of human seborrheic keratoses has been shown, the time point for the occurrence of these mutations is unknown. Therefore, we analyzed whether FGFR3 mutations are already present in flat (initial) seborrheic keratoses and therefore occur early, or are associated with an increased thickness of the tumors and represent therefore late genetic alterations mediating an increased vertical growth of flat seborrheic keratoses. Our results show that FGFR3 mutations are already present in flat seborrheic keratoses. This finding indicates that the mutations may be early genetic events in the pathogenesis of at least a subset of seborrheic keratoses.

Interestingly, we found that the frequency of FGFR3 mutations was significantly associated with an increased age. It has to be noted that the age in this study is defined as the time point of seborrheic keratoses removal and does not necessarily reflect the age of the first appearance of the lesions. However, individuals with FGFR3 mutant seborrheic keratoses were, on average, 10 years older than individuals with wild-type seborrheic keratoses. This result suggests that age is a major risk factor for the occurrence of somatic FGFR3 mutations in skin. One could speculate that cumulative exposure to environmental factors such as chronic UV-light exposure may contribute to FGFR3 mutations in aging skin.

This hypothesis is further supported by the fact that in our study seborrheic keratoses localized on the head and neck revealed a significantly higher FGFR3 mutation frequency than seborrheic keratoses localized on the trunk and extremities, correlating well with a higher cumulative lifetime UV-light exposure of the head and neck compared with the trunk. Furthermore, the R248C mutation was the most frequently detected mutation in our study, particularly in the head region. This mutation is a typical UV-signature mutation. It had been postulated that age and UV light are independent risk factors for the development of seborrheic keratoses. ${ }^{1,2}$ The results of this study suggest that these risk factors contribute not only to the pathogenesis of seborrheic keratoses in general, but particularly to the development of seborrheic keratoses caused by FGFR3 mutations. However, the fact that in some families seborrheic keratoses appear to be autosomal dominant inherited suggests that the genetic background of an individual may be also an important factor for seborrheic keratoses development. ${ }^{.6,17}$
The details of FGFR3 signaling in wild-type and mutant epidermal cells remain elusive. It is supposed that FGFR3 signaling is cell type-specific. ${ }^{18}$ Activating FGFR3 mutations in human skin may provide proliferative signals for the keratinocytes via the Ras-MAPK pathway. Moreover, anti-apoptotic pathways induced by recruitment of PI-3 kinase may result in a prolonged survival of the keratinocytes in FGFR3 mutant seborrheic keratoses. ${ }^{10,19}$ Our immunohistochemical data show that seborrheic keratoses independent of their FGFR3 status reveal a significantly increased cell proliferation compared with normal skin. A previous study reported also an increased Ki-67 expression in seborrheic keratoses compared to normal skin. ${ }^{20}$ Expression of antiapoptotic bcl-2 was significantly associated with FGFR3 mutations in acanthotic seborrheic keratoses, suggesting that the anti-apoptotic PI-3K/Akt-pathway upstream of bcl-2 may be involved in addition to an increased proliferation. Another study found a significantly increased bcl-2 expression in human seborrheic keratoses compared with inflamed seborrheic keratoses and inverted follicular keratoses, but these authors did not investigate the FGFR3 status of the lesions. ${ }^{21}$ We found that FGFR3 mutations were associated with a slightly increased FGFR3 protein expression. This correlation was barely significant, and further studies have to confirm a potential FGFR3 protein overexpression in mutant seborrheic keratoses. FGFR3 mRNA and protein overexpression is known from urothelial carcinoma, and monoclonal antibodies against FGFR3 inhibited the proliferation of an urothelial cancer cell line. ${ }^{22}$ Likewise, FGFR3 protein overexpression in a thyroid cancer cell line led to the loss of cell densitydependent growth inhibition. ${ }^{23}$

While alternative or additional genetic alterations in human seborrheic keratoses as well as details of the involved signaling pathways need to be further determined, the mutant FGFR3 receptor represents a promising pharmaceutical target for the topical treatment of multiple seborrheic keratoses, which are still a major disfiguring problem of aging skin.

\section{Acknowledgements}

We thank Lydia Kuenzel, Kirstin van der Keur, Monika Kerscher, Andrea Müller and Anne Pietryga-Krieger for excellent technical support.

\section{Conflict of interest}

The authors declare no conflict of interest.

\section{References}

1 Yeatman JM, Kilkenny M, Marks R. The prevalence of seborrhoeic keratoses in an Australian population: 
does exposure to sunlight play a part in their frequency? Br J Dermatol 1997;137:411-414.

2 Kwon OS, Hwang EJ, Bae JH, et al Seborrheic keratosis in the Korean males: causative role of sunlight. Photodermatol Photoimmunol Photomed 2003;19: 73-80.

3 Pierson D, Bandel C, Ehrig T, et al Benign epidermal tumors and proliferations. In: Bolognia JL, Jorizzo JL, Rapini RP (eds). Dermatology. Mosby: New York, 2003, pp 1697-1703.

4 Vun Y, De'Ambrosis B, Spelman L, et al Seborrhoeic keratosis and malignancy: collision tumour or malignant transformation? Australas J Dermatol 2006;47: 106-108.

5 Logie A, Dunois-Larde C, Rosty C, et al Activating mutations of the tyrosine kinase receptor FGFR3 are associated with benign skin tumors in mice and humans. Hum Mol Genet 2005;14:1153-1160.

6 Hafner C, van Oers JM, Hartmann A, et al High frequency of FGFR3 mutations in adenoid seborrheic keratoses. J Invest Dermatol 2006;126: 2404-2407.

7 van Oers JM, Lurkin I, van Exsel AJ, et al A simple and fast method for the simultaneous detection of nine fibroblast growth factor receptor 3 mutations in bladder cancer and voided urine. Clin Cancer Res 2005;11:7743-7748.

8 Hafner C, van Oers JM, Vogt T, et al Mosaicism of activating FGFR3 mutations in human skin causes epidermal nevi. J Clin Invest 2006;116:2201-2207.

9 Erkek E, Bozdogan O, Atasoy P, et al BCL-2-related apoptosis markers in cutaneous human papillomavirus-associated lesions. Am J Dermatopathol 2004;26:113-118.

10 Hafner C, Vogt T, Hartmann A. FGFR3 mutations in benign skin tumors. Cell Cycle 2006;5:2723-2728.

11 Hafner C, Hartmann A, Real FX, et al Spectrum of FGFR3 mutations in multiple intraindividual seborrheic keratoses. J Invest Dermatol 2007, E-pub ahead of print [PMID: 17392824].
12 Cappellen D, De Oliveira C, Ricol D, et al Frequent activating mutations of FGFR3 in human bladder and cervix carcinomas. Nat Genet 1999;23:18-20.

13 Vajo Z, Francomano CA, Wilkin DJ. The molecular and genetic basis of fibroblast growth factor receptor 3 disorders: the achondroplasia family of skeletal dysplasias, Muenke craniosynostosis, and Crouzon syndrome with acanthosis nigricans. Endocr Rev 2000; 21:23-39.

14 Passos-Bueno MR, Wilcox WR, Jabs EW, et al Clinical spectrum of fibroblast growth factor receptor mutations. Hum Mutat 1999;14:115-125.

15 Naski MC, Wang Q, Xu J, et al Graded activation of fibroblast growth factor receptor 3 by mutations causing achondroplasia and thanatophoric dysplasia. Nat Genet 1996;13:233-237.

16 Rongioletti F, Corbella L, Rebora A. Multiple familial seborrheic keratoses. Dermatologica 1988;176:43-45.

17 Bedi TR. Familial congenital multiple seborrheic verrucae. Arch Dermatol 1977;113:1441-1442.

18 L'Hote CG, Knowles MA. Cell responses to FGFR3 signalling: growth, differentiation and apoptosis. Exp Cell Res 2005;304:417-431.

19 Eswarakumar VP, Lax I, Schlessinger J. Cellular signaling by fibroblast growth factor receptors. Cytokine Growth Factor Rev 2005;16:139-149.

20 Conscience I, Jovenin N, Coissard C, et al P16 is overexpressed in cutaneous carcinomas located on sun-exposed areas. Eur J Dermatol 2006;16:518-522.

21 Ko CJ, Shintaku P, Binder SW. Comparison of benign keratoses using p53, bcl-1, and bcl-2. J Cutan Pathol 2005;32:356-359.

22 Gomez-Roman JJ, Saenz P, Molina M, et al Fibroblast growth factor receptor 3 is overexpressed in urinary tract carcinomas and modulates the neoplastic cell growth. Clin Cancer Res 2005;11:459-465.

23 Onose H, Emoto N, Sugihara H, et al Overexpression of fibroblast growth factor receptor 3 in a human thyroid carcinoma cell line results in overgrowth of the confluent cultures. Eur J Endocrinol 1999;140:s169-s173. 